\title{
ADVOCACIONES MARIANAS \\ EN LA DOCUMENTACIÓN LEONESA ALTOMEDIEVAL \\ (775-1230)
}

\author{
MARgarita CANTERA MONTENEGRO \\ Universidad Complutense de Madrid \\ CONCEPCIÓN MENDO CARMONA \\ Universidad Complutense de Madrid
}

Este artículo forma parte de un trabajo más amplio, y todavía sin concluir, sobre las advocaciones religiosas en la documentación leonesa altomedieval, que nos encomendó nuestro maestro, el Dr. D. Emilio Sáez, que también lo fue de la fallecida Dra. Sáinz de la Maza.

La documentación utilizada para su realización es fundamentalmente la de la catedral de León ${ }^{1}$, la de Sahagún ${ }^{2}$ y, con menor abundancia de datos, la de San Pedro de Montes ${ }^{3}$.

El estudio de las advocaciones de los monasterios e iglesias es interesante, pues son reflejos claros de las corrientes culturales que confluyen en una zona y una manifestación de la religiosidad de la época.

\footnotetext{
'Colección documental del Archivo de la Catedral de León (775-1230), León, 1987-1991, 6 vols. El primer volumen fue publicado por Emilio SÁEZ; el segundo por Emilio SÁEZ y Carlos SÁEZ; el tercero y cuarto por José Manuel RUIZ ASENCIO; y el quinto y sexto por José $\mathrm{M}^{\mathrm{a}}$ FERNÁNDEZ CATÓN

${ }^{2}$ Colección diplomática del monasterio de Sahagún, León. El primer volumen fue publicado por José Ma MíNGUEZ en 1977; el segundo y tercero por Marta HERRERO, en 1988; y el cuarto por José Antonio FernánDEZ FlórEZ, en 1991.

${ }^{3}$ Augusto Quintana PrIETo, Tumbo Viejo de San Pedro de Montes, León, 1971.

"Anuario de Estudios Medievales", 28 (1998)
} 
El culto a la Madre de Dios es el más importante por el número de iglesias y monasterios a Ella consagrados; y, además, es una de las advocaciones que más pronto aparece en esta documentación. En general, el culto a Santa María es muy temprano en la Iglesia cristiana, sobre todo a partir de la paz que disfruta con Constantino; y así se instituyó una fiesta de la Virgen como reflejo de esa tendencia general de devoción hacia la Madre de Dios. Aunque el culto se desarrolló mucho a partir del Concilio de Éfeso, el 431, la introducción de las fiestas de los misterios de la Virgen parece ser posterior, quizás del siglo VII, momento para el que está bien atestiguado en España con numerosas iglesias dedicadas a Santa María y una solemnísima fiesta que parece indicar un culto más antiguo ${ }^{4}$. A partir de ese momento, Santa María será titular no sólo de capillas privadas e iglesias de monasterios, sino que ya en época visigoda se empieza a dar su nombre a muchas catedrales 5 .

Así ocurre en el caso de la catedral de León, que fue puesta bajo la doble advocación de "Sancte Virginis Marie, regine coelestis, siue uenerande Sancte Zipriani espiscopi", aunque con mucha frecuencia sólo figura bajo el nombre de Santa María, advocación completada a menudo con los títulos de Reina del Cielo y Madre de Dios. En raras ocasiones entre los años 1073 y 1091 se une a las advocaciones de santa María y san Cipriano la de san Salvador, y en una sola ocasión las de san Juan Bautista y san Cristóbal, posiblemente por tener altares consagrados en dicha iglesia. Con frecuencia también se la denomina Sancte Marie Regula o de Regla.

La primera referencia a esta sede catedral es del año 874 , cuando el obispo Frunimio le donó varios objetos litúrgicos y otros bienes, siendo una de las primeras dotaciones de la iglesia principal de la recién reconquistada León. A partir de esa fecha, y ya de modo especial entrado el siglo X, son casi continuas las referencias a este templo de Santa María, unas de forma propia, por ser receptora de donaciones, de forma especial entre los años 916 y 935 en los que Ordoño II y Ramiro II la dotan generosamente, o por tener la iniciativa de realizar alguna compra; y otras por referencias a los obispos de la sede de Santa María en la datación de algún documento; o

${ }^{4}$ Carmen García Rodríguez, El culto de los santos en la España romana y visigoda, Madrid, 1966, pp. 125-126.

5IDEM. ibidem. p. 127. 
incluso por estar cerca de otras iglesias, monasterios o solares de la ciudad de León.

Como es lógico, la iglesia catedral de Santa María y San Cipriano de León está dentro de los muros de la ciudad. Y entre los numerosos monasterios de León hay algunos que estaban especialmente cerca de ese templo: el de San Vicente, el de Santiago, el de los Santos Pedro y Pablo y el de San Juan Bautista, llamado también de Santa María.

Santa María de León es, lógicamente, un templo de gran importancia por el hecho de ser la iglesia catedral de esa ciudad, que pronto se convirtió en la sede de la corte; por ello, los reyes de León eran entronizados en esta iglesia, como dice Alfonso $\mathrm{V}$ el año 1012: "me perduxerunt ad ordinem regali hic in sedem Legione et uocitata eglesie Sancte Marie semper Uirginis", motivo por el cual, y siguiendo la antigua costumbre de sus predecesores, hizo donación a la iglesia del castillo de San Salvador de Curueño.

Otras dos catedrales dedicadas a Santa María figuran en la documentación consultada: la de Astorga, desde mediados del siglo XI, y que es citada con frecuencia en los diplomas de San Pedro de Montes, al recoger el nombre de su obipo como elemento de datación o como confirmante de algún documento. Y la de Toledo, citada en las mismas circunstancias desde el año 1087.

Como ya hemos dicho, son muy numerosos los monasterios de la documentación leonesa que tienen a santa María como titular. Uno de los más importantes es el de Santa María y Santiago de Valdevimbre; no se puede precisar cuál de las dos advocaciones es la principal en los primeros momentos, ya que suelen ir unidas; también vemos aparecer en bastantes ocasiones, y casi con la misma frecuencia, sólo una de las dos, ya sea la mariana, ya la del apóstol. Las citas documentales de Santa María empiezan en el año 937, siendo más tempranas las de Santiago (año 915); las dos permanecen hasta 1050, fecha a partir de la cual sólo figura Santa María, manteniéndose en solitario esta advocación en 1116 cuando el cenobio es donado, a la sede de León por el obispo don Diego.

También Santa María aparece como advocación conjunta de otro monasterio, el de Santiago de León, monasterio dúplice enclavado dentro de la ciudad. En este caso, Santa María es la advocación secundaria, ya que es más frecuente la referencia exclusiva al apóstol; y sólo en dos ocasiones se habla de monasterio de Santa María sin mencionar a Santiago. 
Otro importante monasterio acogido a la advocación mariana es el de Piasca, en Liébana, que figura en la documentación de Sahagún desde el año 930. En los primeros años, figura también entre los titulares, aunque en contadas ocasiones, Santiago, san Juan apóstol y los santos Julián y Basilisa; y en 1030, san Pelayo y san Juan Bautista.

Se trata de un monasterio dúplice, regido en ocasiones por una abadesa. Casi siglo y medio después de la primera cita del cenobio, en 1075, nos encontramos un documento por el cual la condesa Urraca, Christi ancilla, y los descendientes de los condes Munio, Gutier y Adosinda se comprometían a no dividir por herencia este monasterio, al tiempo que establecían que la comunidad podía elegir por señor a un miembro de la familia, que sería quien nombraría abad; señalaban, además, la obligación de los monjes de acoger, en caso de vejez o enfermedad, a los miembros de dicha familia que así lo desearan. A pesar de la obligación de mantener indiviso el monasterio, entre los años 1080 y 1108 son varios los documentos del cenobio de Sahagún por los cuales diversas personas, seguramente miembros de esa misma familia, donaban al cenobio leonés la porción que les pertenecía en ese centro, dependencia que sería confirmada en 1163 y 1194 por Alejandro III y Celestino III, respectivamente.

También recibió la advocación de Santa María una de las iglesiaseremitorios de Parameno o Pardomino, y que figura en la documentación entre los años 954 a 1013.

En la segunda mitad del siglo XII, en 1169, fue fundado el monasterio de Santa María de Gradefés, de monjas cistercienses. Y desde esa fecha aparece con frecuencia en documentos relacionados con la formación de su dominio.

Otros dos monasterios famosos figuran en esta documentación, aunque de forma marginal: el de Santa María de Bamba, seguramente el famoso prebenedictino de Valladolid, cuyo abad confirmó, a mediados del siglo X, varias donaciones reales a Sahagún; vuelve a aparecer en la segunda mitad del XII como lugar elegido por Rodrigo Pelagii para ser enterrado, aunque ahora como iglesia. El otro es el cisterciense de Moreruela, que aparece en 1189 en la documentación de Sahagún como destinatario de una donación.

Aparte de estos monasterios más destacados, son otros varios los que se titulan de Santa María. De muchos de ellos tenemos sólo una única y escueta cita, para no volver a saber nada de ellos; es posible, por tanto, que se tratase de pequeños monasterios familiares que, con el paso del tiempo, 
acabarían desapareciendo o integrándose en otro monasterio o iglesia más importante. A veces es precisamente este acontecimiento, su donación a otro centro eclesiástico, el que nos transmite la noticia de su existencia. Es el caso del monasterio situado in territorio Tinegio, junto al río Arlanza, que Alfonso III entregó a la iglesia de San Salvador de Oviedo el 906, con su villa y propiedades, sus decanías situadas en Galicia, junto al Miño, y algunos otros bienes. Y lo mismo ocurre con los cuatro monasterios que el 1037 recibió la iglesia catedral de León donadas por Elvira, hija del conde Fáfila Fernández. Todos están situados en el norte, en las actuales provincias de Asturias y Santander: uno en Lebeña y otro en "Vejo" (Bellio); los otros dos llevan advocación conjunta: San Pedro, San Julián y Santa María, en el valle de Bedoya; y el de Santa María y San Tirso, en rivulo de Obles.

Son varios los monasterios bajo advocación mariana que se integraron en el de Sahagún: el de Oseja de Sajambre, en territorio de Riaño y junto al río Sella, se incorporó a este cenobio en 1028 por medio de una permuta de bienes, aunque hay una referencia anterior, del 1005, como destinatario de una donación, figurando en esta ocasión también bajo la advocación de los Santos Facundo y Primitivo. El de Curueño aparece por primera vez en la documentación en 1055, con motivo de una donación; y a la advocación mariana principal se unen Santiago, San Pelayo, San Bartolomé y San Mamés; aunque no sabemos cuándo se incorporó a Sahagún, le fue confirmado por Celestino III a fines del siglo XII. En 1064 Osorio Osóriz donó el de Páramo, situado junto al río Carrión, entre Calzada y Torre. El de Villaturde lo donó la condesa Momadonna en 1071, con todo lo suyo y sus hombres. Cuatro años más tarde, Fernando Pérez y su mujer Elvira donaron el de Egunna, posiblemente en Boñar. El de Villadevande fue donado en dos partes, en 1077 y 1094, por el abad Aiub y por Pedro Vellítiz. En 1091 fueron donados otros dos cenobios: el de Escaro, en territorio de Burón, por parte del presbítero Flaíno; y el de Villafilal, por María Muñoz y sus hijos. Dos años más tarde se incorporaron otros dos: el de Cabarroso, en el alfoz de Saldaña, donado por Alfonso VI junto al monasterio de San Salvador de Nogal, al que pertenecía; y el de "Villa Ramelle" (seguramente Villarramiel), construido por el propio donante, el presbítero Ariulfo, que recibió el apelativo de monasteriolo, figurando también en advocación conjunta con la Santísima Trinidad. Por último, en 1125 fue donado el de "Feleyta". También pertenecía a Sahagún el de Santa María de Pozadurana, que en 1096 fue entregado a los hijos de Monio Velázquez a cambio de la propiedad que éstos tenían en el monasterio 
de San Felices. Hay otro monasterio familiar vinculado en 1078 al de Sahagún, pero del que no se da ninguna referencia geográfica para poder localizarlo; a pesar de ello creemos que no puede identificarse con ninguno de los otros ya recogidos en este apartado. Por último, el cenobio de Sahagún recibió el año 1186 la donación del monasterio de San Pelayo de Pedrezangas y con él se entregaba también el de Santa María de "Amaiolas", posiblemente un pequeño monasterio o quizás una iglesia, ya que no se especifica nada más.

También a la catedral de León pertenecieron algunos cenobios dedicados a la Madre de Dios, como el de "Villa Abarca" que en 1077 el obispo de León entregó a un particular con condición de que lo devolviera a su muerte. O el de los Santos Pedro y Pablo y Santa María en Alcuetas, donado por sus edificadores, Pedro Móniz, su mujer e hijos.

Por último, el de Santa María de Carbajal: fue donado en 1144 por don Juan, obispo de León, y los canónigos de esa sede, como lugar de retiro para los canónigos que desearan una vida más estricta; estaba bajo la dependencia jurídica del obispo y seguía la Regla de san Agustín. Poco después se transformó en abadía femenina ${ }^{6}$, y como tal figura en alguna otra ocasión.

Otros cenobios vieron incorporar en sus dominios algún monasterio mariano. Es el caso del de San Salvador de Cantamuda, que recibió en 1069 el de "Flavenia", en Lebeña. El de Piasca recibió uno en Perrozo el año 1030. Al de San Mancio se incorporaron uno en Escobar y otro posiblemente en Espinosa. Al de San Salvador de Nogal, uno en territorio de San Román de Entrepeñas y otro en la villa llamada Serna. Y, por último, al de San Martín de la Fuente el de "Valdetolo", en 1048.

De algunos otros monasterios es bien poco lo que sabemos. En Saelices de Mayorga dotó Ordoño II, en 921, un monasterio dedicado a San Félix, Santa María y Santos Pedro y Pablo. Del de "Pedonis" sólo tenemos una referencia del año 934, por ser limítrofe con una tierra vendida al cenobio de Sahagún. Y del de Piniares, en Liébana, que en 997 permutó ciertas propiedades. En Valderrueda, junto al Cea, está el monasterio de Santa María que recibió en 1062 todos los bienes que poseía el presbítero Ansur Andolfiz. En 1175 se habla del monasterio de Santa María de Caridad, como perteneciente al obispado de Ciudad Rodrigo. Entre 1181 y

${ }^{6}$ Diccionario de Historia Eclesiástica de España, III, p. 1541. 
1186, el de Santa María de los Valles, de Peñacorada, recibió algunas donaciones, dos de ellas del monarca leonés Fernando II. Por último, el abad del monasterio de Santa María de Arbás sostenía un pleito con Diego Roderici y su mujer, sobre el derecho de patronato que ésta alegaba en la iglesia de Santa Doradia, en la diócesis de Oviedo.

Tenemos sólo un documento de otro monasterio de Santa María, cenobio femenino, y cuya abadesa, en 999, vendió el monasterio de Santiago de León ciertas propiedades en el "suburbium" de la ciudad, cerca del río Bernesga y lindando con un canal que llevaba agua del Torío. Estas son las únicas referencias geográficas que aparecen en este documento, referencias escasas pero que permiten no identificar el monasterio con ninguno de los otros. Tampoco se puede confundir con una iglesia, ya que se habla claramente de domna Maria abadissa una pariter cum collegio Sancte Marie.

Poco anterior es la noticia de otro monasterio, San Esteban, San Martín y Santa María de Corniero, que recibe una donación de unos particulares. Aunque se habla de iglesia, creemos que se trataría de un monasterio familiar, y quizás dúplice, ya que recibe una donación a favor de dicha iglesia y de doña Jimena, realizada por dos "fratreres" y sus "ad gultores".

Poco más numerosas son las referencias al monasterio de Manzaneda, cerca del río Torío, y que el obispo de León Froilán II entregó a la sede catedral en 1002 .

Más compleja es la referencia a un monasterio de Santa María situado en la ciudad de León y que posiblemente no sería un monasterio propiamente dicho, sino una iglesia dentro del monasterio de San Juan Bautista de dicha ciudad: el año 1011 el conde Munio Fernández y su mujer, doña Elvira, fundaron y dotaron dicho monasterio del Bautista, entregando para ello un solar cerca de la Puerta de Arco de Rey, donde existían dos torres; y, una vez terminado, quisieron erigir en una de dichas torres, la situada al oriente, una iglesia con reliquias del Lignum Crucis, de los santos Pedro y Pablo y de san Juan Bautista, et allia ecclesie Sancte Marie. Posiblemente esta iglesia ganaría importancia con el tiempo, y por ello en 1030 y 1049 se habla del monasterio de San Juan y Santa María de León, por unir ambas advocaciones. 
También en la ciudad de León hay otro monasterio acogido a la protección mariana, y diferente del anterior. Se trata, según Risco ${ }^{7}$ de un monasterio dúplice construido dentro de los muros de la ciudad y que fue fundado por el infante don Ordoño, hijo bastardo de Vermudo II, y su mujer Fronilde.

Aunque este autor retrasa la primera referencia documental del monasterio al año 1042, año de la dotación por el infante, afirma a continuación que un diploma del 1037 revela su existencia en esa fecha; este documento es la donación que el presbíerto Ecta hizo al citado monasterio de la mitad de la villa de Matallana. El documento puede hacer dudar un poco del carácter monástico del centro, ya que habla en dos ocasiones de "aula", es decir, iglesia; pero también lo hace de "monasteriolo" y, más significativo aún, de "homnem collegio". El presbítero justifica esta donación, que dispone en sus últimas voluntades, porque había permanecido durante mucho tiempo en dicho monasterio, antes de servir al conde Fernando Flaínez; el hecho de que el presbítero nombrara como albaceas al abad Muza y a doña Gotina, pidiendo que su cuerpo fuera enterrado en la iglesia del monasterio, avala la afirmación de Risco de que se trataba de un monasterio dúplice ${ }^{8}$. Y a este monasterio se refiere otro documento, posiblemente del año 1035, y que vuelve a ratificar su carácter dúplice, pues está regido por una abadesa, que realiza una permuta de propiedades; en este documento se afirma que la norma de vida y disciplina del monasterio era la Regla de San Benito.

Hay otro monasterio mariano, femenino, en la localidad de Navatejera, cuyas monjas eran las propietarias de una viña vendida entre particulares a principios del siglo XI.

Para terminar este apartado de los monasterios dedicados a la Madre de Dios hay que referirse a varios cenobios en los que Santa María figura en lugar menos destacado junto a otras advocaciones, como el de San Antonino o Antolín, cerca de Coyanza; el de San Martín de la Fuente; el de los Santos Facundo y Primitivo, San Félix, Santa María y Santos Pedro y Pablo en territorio de Melgar, junto al Cea; el de San Salvador de Matallana; el de San Salvador de Villacete; el de San Salvador de Cantamuda; el de

\footnotetext{
${ }^{7}$ Manuel Risco, Iglesia de León y monasterios antiguos y modernos de la misma ciudad, Madrid, 1792, p. 132.

${ }^{8}$ Hay, además, una referencia posterior, del año 1049, a monagos et sorores.
} 
San Salvador y Santa María de Sahagún; y el de San Pedro de Montes, aunque en muy pocas ocasiones. En esta relación se puede observar que con mucha frecuencia se unen la advocación mariana y la del Salvador, Madre e Hijo unidos profundamente en la religiosidad popular.

Si numerosos son los monasterios bajo la advocación de Santa María, más son todavía las iglesias, de muchas de las cuales apenas tenemos más que una o dos referencias y, a menudo, imprecisas o vagas. La primera es una "baselica" situada junto al río Aller, al sur de la actual provincia de Asturias, y que el año 860 fue donada por el monarca Ordoño I al obispo Frunimio.

En la misma región está la basílica de Santa María y Santa Marina, en el valle de Caso y junto al río Velerda (afluente del Nalón), que en el año 895 recibió del presbítero Seovano una basílica dedicada a san Martín, en el territorio de Noanca. Esta iglesia, años más tarde, figura sólo bajo la advocación de Santa María.

Nueve años más tarde se habla de otra iglesia de Santa María, enclavada en el suburbio, o las afueras, del Castro de Monzón; esta iglesia, según se narra en el documento, fue destruida por los musulmanes (paganis) y restaurada por el presbítero Gratón, quien la donó en este momento a su señor, el diácono e infante Gonzalo.

Hay una serie de iglesias que fueron donadas por los reyes: el 906, Alfonso III entregó a San Salvador de Oviedo una iglesia dedicada a Santa María en el Castro de Coyanca. Más numerosas son las donadas por Ordoño II a la sede legionense en el año 916. En abril entregó una en Araduey; otra a orillas del Porma, sin más detalles; y las llamadas de Abarca, de Carrión, de Foro y de Palacio, las dos últimas situadas entre los ríos Eo y Masma, en la actual provincia de Lugo y cerca de Asturias. En esta misma región estaban las otras cuatro iglesias de Santa María donadas por el mismo rey en diciembre: la de Tabulata, de Osorici, de Ferrarios y de Iconi, cuya posesión fue confirmada a Santa María de León unos años después, en 935, por Ramiro II (excepto la de Iconi).

Dos años más tarde, en 918, el mismo monarca realizó una nueva donación a la catedral de León y entre los bienes entregados hay otras dos iglesias dedicadas a Santa María, una en "Vieira" y la otra junto al Bernesga; en esta última, la advocación mariana figura como secundaria, por ser san Félix la primera.

Hay otras dos iglesias dedicadas a Santa María junto al río Cea. Una de ellas figura en 917 y 944 como objeto de donación al monasterio de 
Santiago de León. Sorprende un poco el hecho de que, siendo clara la donación en el primer documento, ésta se repita, 27 años después; posiblemente se explica esta repetición porque la primera donación no se pudiese hacer efectiva, o incluso porque volviese a manos de particulares, pues el abad Cipriano, protagonista de la donación del 944, declara haberla comprado a un tal Nuño, ya fallecido.

La otra iglesia de Santa María, situada también a orillas del Cea, sí puede plantear la duda de si se tratará de la misma de los documentos anteriores o de otra distinta; esta iglesia, de la que se especifica que está sobre Castrobol, fue donada el año 948 por el obispo Oveco de León al abad de San Cipriano del Condado y a la abadesa de Santiago de León, y a sus respectivas comunidades, justificando que podía hacer esta donación porque las institutciones canónicas permitían al obispo parrocianis eclesiis monasterium facere. Aunque pueda haber alguna duda sobre su posible identificación con la otra iglesia de Santa María junto al Cea, creemos casi con total seguridad que se trata de otra diferente: si podemos admitir la necesidad de "reiterar" la donación anterior al cabo de un tiempo, quizás por no haberse hecho efectiva, sería muy extraño que tuviera que ratificarse sólo cuatro años después de la segunda donación, además compartiendo la propiedad con otro monasterio; por otra parte, el especificar ahora su localización junto a Castrobol, y no haberlo hecho en los otros documentos, parece confirmar la idea de que se trata de dos iglesias diferentes. Casi un siglo después, el año 1042, el infante Ordoño y su mujer Fronilde dotaron el monasterio de Santa María de León, como ya hemos dicho, y entre los bienes entregados se encontraba la iglesia de Santa María de Castrobol; en este caso, creemos que se trata de la misma iglesia que posiblemente, había llegado al patrimonio del infante por medios justos (compra o permuta) o por usurpación en ese siglo que media entre la primera y la segunda donaciones.

De algunas iglesias dedicadas a Santa María tenemos sólo la noticia de su dependencia o incorporación a otro centro eclesiástico de mayor importancia. Respecto a la catedral de León, en primer lugar sabemos de cinco iglesias enclavadas en Liébana y que siendo obispo de esa sede don Alvito, a mediados del siglo XI, entregaban cierta renta a la sede catedral: son la de Cosgaya, la de Prado, la de Roval, la de Varaio y la de Vegio. La de Roales fue donada, en 1076, por el obispo don Pelayo con motivo de su consagración.

En 1095 la catedral recibió la iglesia de Santa María Alba, siendo los donantes los concejos que acudían a esa iglesia; este lugar acabará con el 
nombre de Marialba, un claro ejemplo de hagiotopónimo. Esta iglesia ya existía en el año 976; y casi un siglo después de la primera donación a la catedral de León, fue donada de nuevo a la misma sede por el rey Fernando II y su hijo Alfonso [IX], lo que hace pensar que habría sido usurpada a la catedral.

Para iluminar los altares de Santa María de León entregó el obispo de esta sede en 1120 la iglesia de Santa María de Vico Francorum, cuyos capellanes se comprometían a entregar anualmente a la primera un tercio de los diezmos, ofrendas y mandas de los difuntos. También recibió la iglesia fundada en el castillo de Valderas, en 1142, siendo los donantes los propios edificadores de esa iglesia. Santa María de Mercado, en Mayorga, fue donada en 1157, reservándose el donante, Martín Pelagii, el usufructo de ella mientras vivía; años más tarde, en 1186 el obispo de León concedió que las tercias de esta iglesia, durante diez años, se utilizasen, junto con otros ingresos, para la fábrica y ornamentos de las iglesias de la localidad de Mayorga. Fernando II fue el donante de la de Valdemora, el año 1164. En 1187, el mismo monarca confirmó la posesión de la iglesia de Santa María en Castroverde (de Campos), situada junto al castillo y que había sido donada años atrás, en 1169 , por varios particulares.

Los derechos sobre algunas iglesias plantearon problemas entre la sede de León y otras entidades. Así, la de Mansilla de las Mulas, acogida a la advocación mariana, hasta que en 1182 las dos partes, obispo y canónigos, por una parte, y habitantes y clérigos de esa localidad, por la otra, llegaron a un acuerdo sobre los derechos de diezmos y tercias, que pertenecían a la iglesia de León, que también sería la encargada de nombrar el clérigo que la cuidaría; a pesar de ello, debieron de seguir las diferencias, pues el papa Inocencio III nombró, en 1205 una comisión para estudiar el asunto. La iglesia de Santa María del Azogue fue también objeto de un acuerdo entre el obipo y Pedro Monacino, quien renunció a la iglesia a favor del obispo, a cambio de disfrutar de ella mientras viviese. Los derechos en la de Chanos, quizás Llanos, fueron renovados en 1210. Y, por último, la de Villada era objeto de pleito entre la catedral y el monasterio de Sahagún, declarando un testigo en 1215 que pertenecía al obispo.

Varias son las iglesias de Santa María que eran propiedad del monasterio de Sahagún: la primera de ellas fue donada a ese cenobio el año 922 y confirmada tres años más tarde; pero no se da ninguna referencia geográfica de ella, por lo que es imposible localizarla. Otra estaba en Valle nuncupato Ratarii, y fue donada el 962 por el obispo de León don Gonzalo. 
La de Curueño fue entregada por el monasterio de Sahagún al de Santiago de León en una permuta el año 980; casi un siglo después se le da la advocación conjunta de Santa María, Santiago, San Pelayo, San Bartolomé y San Mamés. En 1037 fue donada la de "Requexolo", junto al río Valderaduey. Y al año siguiente la de Galleguillos, junto al río Cea. La de Santa María del Valle, cerca de Saldaña, fue donada en 1148 por Alfonso VII y entregada años más tarde a la abadesa Mayor para que la tuviera mientras viviese; esta entrega hace pensar que se trataría de un monasterio, como se le llama en el primer documento, pero fue confirmada por Celestino III en 1194 como iglesia, lo que indicaría su transformación en el proceso de reforma eclesiástica iniciada en el siglo XI. Este mismo pontífice confirmó, en ese mismo documento, otras tres iglesias dedicadas a la Madre de Dios, la de Villavicencio, la de "Yevas" y la de Villafrades, esta última en el obispado de Palencia. Y, por último, en 1186 recibió una parte de la iglesia de Santa María en Villagómez.

En el patrimonio de San Pedro de Montes hay también varias iglesias dedicadas a la Madre de Dios: la de Casayo, donada al monasterio en 1075. La de Jagoaza de Valdeorras, en el año 1092. La de La Ermida, en Jagoaza y cerca del río Sil, fue donada, o al menos alguna parte de ella, en 1096, el mismo año que lo fue la de Voces. A mediados del siglo XII se integraron en el dominio monástico dos quintas partes de la iglesia de Santa María de Villanueva de Valdueza, y el resto debió de incorporarse después, ya que a pincipios del siglo XII el papa Inocencio III confirmó dicha iglesia al monasterio de San Pedro; de todas formas, la primera cita de esta iglesia es del 1093. En Palacios de Rey está otra iglesia de Santa María, donada en este caso por Alfonso IX. Un documento de Inocencio III confirma a este cenobio la propiedad de otras cinco iglesias de Santa María: la de Cabañeros, la de Castrello de Valdueza, la de Villafranca, la de Orres (quizás Valdeorras) y la de Ecclesia Rubie. Por último, sabemos que también tenía ciertos derechos en la de Santa María de Escalios o Val de Escalios, según un acuerdo entre el monasterio y el cabildo de Astorga del año 1128.

Al monasterio de San Salvador de Nogal, incluido en el patrimonio de Sahagún, pertenecen también varias iglesias dedicadas a Santa María: las de Gozón, Olmillos en la ribera del Carrión y de Populatione de Iuso, donadas por Alfonso VI el año 1093; y la de Villa Onego, donada tres años después. El monasterio de Santiago de Cellariolo recibió en 975 la iglesia de Santa María, situada en el valle llamado Aureliana; este topónimo quizás podría identificarse con la localidad de Orellán, cerca de Ponferrada. Entre 
994 y 1001 se habla de una iglesia de Santa María situada junto illa penna que dicunt Pombario, iglesia que había pertenecido al obispado de León y que en ese momento era donada por el obispo Froilán al monasterio de los Santos Cosme y Damián de Abeliar. Al de San Martín de Valdepueblo pertenecía la de Santa María de Golpejones, término de Castroverde del Campo (provincia de Zamora), iglesia propia construida en 1025 por Faleón Amátez y su mujer, quienes la donaron a ese cenobio a su muerte. En 1069 el monasterio de San Salvador y Santa María de Cantamuda recibió la iglesia de Santa María de Vejo. Y en 1186, el de Santa María de los Valles recibió de Fernando II de León la de Almoy.

De algunas iglesias dedicadas a la Madre de Dios tenemos sólo una vaga referencia, por ser límite de alguna propiedad. Es el caso de: Santa María junto al río Sequillo; Santa María en Eslonza; la situada seguramente en Valdesaz de los Oteros (Oterolo); la de Tendal, cerca de León; la de Santa María y San Pelayo en Valle, en la región de Valdueza; la de Campo, en el Bierzo; la de Otarelo de Valdeorras, junto al río Sil; la de Nogar de Cabrera; y Santa María de Vega.

También son unas escuetas referencias geográficas las noticias sobre otras iglesias de Santa María, pues en ellas se redactaron diversos documentos: la de Otero, dedicada a san Clemente y santa María; y la de Quintanilla del Monte.

Queda, por último, referirse a varias iglesias de Santa María y de distinta importancia. La de Santa María del Camino figura en varias ocasiones en la documentación de la catedral de León como receptora de donaciones, a partir de 1122; esta advocación refleja claramente que su nacimiento está en relación con el Camino de Santiago, hecho avalado por la presencia de francos vinculados a dicha iglesia ya en la primera cita. La iglesia canonical de Trianos también se acogía a la advocación mariana a fines del XII, cuando fue declarada exenta de la jurisdicción del obispo. La de Villota recibió en 1191 una donación de San Salvador de Nogal. Y un tercio de la iglesia de Santa María en Arcahueja, entre León y Mansilla, fue vendida entre particulares a principios del XIII.

Hay que cerrar este trabajo refiriéndonos a dos hospitales, el de Arvis y el de León, que figuran entre fines del XII y principios del XIII en la documentación de la catedral leonesa. Y, por último, una cofradía dedicada a la Purificación de Santa María en 1212, que nos refleja la profunda devoción a la Madre de Dios y su arraigo popular. 


\section{RÉSUMÉ}

Cet article est une partie d'un travail beaucoup plus long sur les vocables sous lesquels sont les monastères et les églises que nous trouvons dans les documents de León du Haut Moyen Âge. Parmi ces vocables, nous signalons comme le plus important, non seulement par le nombre mais aussi par l'importance des centres religieux, celui de Sainte Marie, témoignage de la dévotion à la Sainte Vierge si charactéristique de la chrétienté médiévale.

\section{SUMMARY}

This paper is part of a larger study of the dedications of monasteries and churches as found in leonese documents of the High Middle Ages. Dedications to St. Mary are among the most numerous and are frequently encountered at the most important of ecclesiastical centres, reflecting the profound marianism characteristic of Medieval christianity. 